\title{
Análise dos processos fonológicos em crianças com desenvolvimento fonológico normal
}

\section{Phonological processes analysis in children with normal phonological development}

\author{
Carla Ferrante ${ }^{1}$, John Van Borsel², Mônica Medeiros de Britto Pereira ${ }^{3}$
}

\begin{abstract}
RESUMO
Objetivo: O presente estudo teve como objetivo verificar o uso dos processos fonológicos em uma população de crianças com desenvolvimento fonológico normal. Métodos: Fizeram parte da pesquisa 240 crianças, de ambos os sexos, com idades entre três e oito anos. Foram realizadas análises relativas aos processos fonológicos e os dados foram comparados em relação à faixa etária e sexo. Resultados: A análise dos resultados permitiu concluir que aos três, quatro e cinco anos os processos de redução de encontro consonantal, lateralização e apagamento de consoante final foram os mais utilizados. A metátese foi o segundo processo mais utilizado na faixa etária de seis anos, aparecendo em terceiro lugar na faixa etária de sete anos. Em relação ao número de processos fonológicos utilizados por faixa etária, aos três anos de idade as crianças utilizaram um mínimo de dois processos e a partir da faixa etária de quatro anos o número mínimo de processos fonológicos utilizados foi zero e o número máximo diminuiu gradativamente de acordo com o aumento da faixa etária, assim como a média. Em relação à variável sexo, não foi observada nenhuma diferença estatisticamente significante em nenhuma das análises realizadas nesta pesquisa. Conclusões: Os dados encontrados nesta pesquisa evidenciam a dificuldade encontrada pelas crianças na produção das líquidas e nas estruturas silábicas mais complexas.
\end{abstract}

Descritores: Linguagem infantil; Avaliação; Desenvolvimento infantil; Desenvolvimento da linguagem

\section{INTRODUÇÃO}

O surgimento da linguagem expressiva ocorre durante os primeiros anos de vida da criança, período em que os fonemas são adquiridos e estabelecidos quanto às posições nas sílabas e nas palavras e de acordo com uma cronologia que é, ao que parece, similar para a maioria das crianças ${ }^{(1)}$. Durante a aquisição fonológica as crianças devem aprender quais os sons usados na sua língua e de que maneira eles são organizados ${ }^{(2)}$. A fase de maior expansão do sistema fonológico ocorre entre 1:6 e 4:0 anos, quando há um aumento do inventário fonético das crianças possibilitando a produção de palavras polissilábicas e de estruturas silábicas mais complexas, porém, este período é caracterizado pela ocorrência de substituições e omissões de sons ${ }^{(3)}$.

A aquisição do sistema fonológico de uma língua, incluin-

Trabalho realizado na Universidade Veiga de Almeida - UVA - Rio de Janeiro (RJ), Brasil.

(1) Mestre; Professora visitante da Associação Pestalozzi de Niterói - ASPNNiterói (RJ), Brasil.

(2) Doutor, Professor Titular da Ghent University - UGent - Zwijnaarde (Gent), Belgium.

(3) Doutora, Professora Adjunto da Universidade Veiga de Almeida - UVA - Rio de Janeiro (RJ), Brasil.

Endereço para correspondência: Carla Ferrante. R. Ipanema, 197, apto 1301, Barra da Tijuca, Rio de Janeiro - RJ, CEP 22631-390.

E-mail: caferrante@ rjnet.com.br

Recebido em: 30/8/2007; Aceito em: 20/10/2008 do seu inventário fonético e as regras fonológicas, ocorre de forma contínua e gradativa até os sete anos ${ }^{(3)}$, implicando na aquisição de um sistema de sons profundamente ligado ao crescimento global da criança em relação ao idioma. Embora seja possível identificar tendências gerais, cada criança desenvolve sua linguagem de forma particular ${ }^{(4)}$.

O distúrbio articulatório, caracterizado pela alteração dos níveis fonético e/ou fonológico da linguagem, é um dos distúrbios fonoaudiológicos de maior ocorrência na população infantil ${ }^{(5)}$ podendo prejudicar os aspectos sociais e escolares da vida da criança, se não diagnosticado e tratado no tempo adequado.

Os processos fonológicos constituem mudanças sistemáticas que afetam uma classe ou sequência de sons e se constituem em descrições de padrões que ocorrem regularmente na fala da criança com o objetivo de simplificar os alvos dos adultos ${ }^{(4)}$. Os processos fonológicos, também chamados de estratégias de reparo, podem ser classificados em três grupos: de estrutura silábica $^{(6)}$, de substituição e de assimilação $0^{(7-8)}$.

Em pesquisa realizada com 134 crianças com desenvolvimento fonológico normal com idades entre 1:0 e 5:3 foi observado que as estratégias de reparo utilizadas em relação à aquisição do onset complexo foram: redução do encontro consonantal, lateralização, metátese, semivocalização, substituição da plosiva e epêntese. As crianças desta pesquisa utilizaram consistentemente o processo fonológico de redução 
de encontro consonantal tanto nos encontros compostos de líquida lateral como nos de líquida não-lateral. Este processo correspondeu a aproximadamente $40 \%$ das estratégias de reparo observadas. Concluiu-se que as estratégias de reparo são utilizadas de maneira distinta pelas crianças mais novas em relação às mais velhas e que a variedade e a quantidade de estratégias empregadas são geralmente maiores entre os sujeitos com idades entre 1:0 e 3:0, ficando mais restritas e menos usadas nos sujeitos entre $3: 2$ e 5:3(9). A ocorrência de metátese se deu majoritariamente entre as crianças mais velhas, o que seria explicado como uma estratégia das crianças que já superaram em parte, ou que ainda estão superando, dificuldades de estrutura silábica e por isso não apagam, mas transpõem os componentes dessas estruturas numa tática de dupla evitação: evitação de estrutura problemática e evitação do apagamento puro e simples. O envolvimento frequente da líquida neste processo ocorre devido ao fato de que esses sons integram as estruturas silábicas mais complexas do português, isto é, CCV e CVC. Além disso, são sons de aquisição tardia e, portanto só poderão estar envolvidos em processos realizados por crianças maiores. Entende-se que a relevância do envolvimento do $/ \mathrm{r} /$ nos processos de metátese está diretamente associada à estrutura silábica complexa em que esse som quase sempre aparece e não à época de aquisição de um ou de outro segmento qualquer ${ }^{(10)}$.

Em outro estudo foi observado que quando os fonemas fricativos não são produzidos corretamente, duas estratégias de reparo são utilizadas: omissão ou substituição do segmento ${ }^{(11-12)}$.

Alguns processos fonológicos costumam desaparecer antes dos três anos de idade (apagamento da sílaba átona, apagamento da consoante final, reduplicação, anteriorização e assimilação) enquanto outros só desaparecem após esta idade, como é o caso da redução de encontro consonantal, epêntese, semivocalização, plosivização e despalatalização ${ }^{(13)}$.

Em crianças falantes do inglês os processos fonológicos encontrados na faixa etária de 3:0 a 3:11 anos foram: redução de encontro consonantal, apagamento de consoante final, apagamento de sílaba átona, anteriorização, plosivização, semivocalização e assimilação. Na faixa etária de quatro anos foram encontrados apenas os processos de redução de encontro consonantal, apagamento de sílaba átona e semivocalização. $\mathrm{Na}$ faixa etária de cinco anos os processos fonológicos utilizados foram epêntese, metátese e desafricação. A partir dos seis anos, as crianças com desenvolvimento fonológico normal não fazem mais uso de processos fonológicos ${ }^{(14)}$.

Pesquisa sobre a produtividade dos processos fonológicos observou que a idade prevista para a eliminação dos processos de apagamento de sílaba, assimilação, plosivização de fricativas, posteriorização e anteriorização de velares e simplificação de líquidas é a de 2:0 a 3:6 anos. Aos 4:6 anos ocorre a eliminação dos processos de posteriorização e anteriorização de palatal. As autoras relatam que sete anos é a idade prevista para a eliminação do uso dos processos de redução de encontro consonantal e apagamento de consoante final pelas crianças com desenvolvimento normal ${ }^{(15)}$.

Outra pesquisa envolvendo 20 crianças com diagnóstico de transtorno fonológico observou grande variabilidade dos processos entre os sujeitos, sendo a redução de encontro consonantal, o ensurdecimento de fricativas e a simplificação de líquidas, os que mais variaram. De uma forma geral, os processos com maior porcentagem de ocorrência foram os que apresentaram maior desvio padrão, o que sugere a existência de diferenças entre os sujeitos quanto ao uso dos mesmos. Nesta pesquisa, foi observado ainda, que o processo de simplificação de líquidas foi comum a todos os sujeitos. Os processos de redução de encontro consonantal, ensurdecimento de fricativas e anteriorização foram usados apenas por parte dos sujeitos, enquanto a posteriorização não foi observado por nenhum dos sujeitos ${ }^{(16)}$. Em outro estudo, também com crianças com transtorno fonológico ${ }^{(17)}$, que buscou investigar a influência do histórico familial, observou-se que simplificação de líquidas foi o processo de maior ocorrência independentemente do histórico familial.

A audição é sem duvida um fator muito importante na aquisição fonológica. Em um estudo com crianças com transtorno fonológico ${ }^{(18)}$ foi pesquisado a ocorrência de processos fonológicos em crianças com e sem histórico de otite média. $\mathrm{O}$ estudo encontrou no grupo de crianças com otite média maior ocorrência dos processos de ensurdecimento de fricativas e plosivas e simplificação de líquidas e no grupo de crianças sem otite os processos que mais ocorreram foram os de simplificação de encontro consonantal, ensurdecimento de fricativas e plosivas e simplificação de líquidas. O estudo não conseguiu estipular marcadores lingüísticos que pudessem diferenciar os dois grupos. A mesma autora, em outro estudo ${ }^{(19)}$ também com crianças com transtorno fonológico, buscou verificar a adequação das provas de imitação e nomeação para deteç̧ão do grau de severidade do transtorno e observou que os processos mais produtivos foram simplificação do encontro consonantal, simplificação de líquidas e eliminação da consoante final.

A alimentação é também um fator importante no processo de aquisição dos sons. Em estudo ${ }^{(20)}$ conduzido com crianças de dois a seis anos, com antecedentes de desnutrição, observou-se atraso na eliminação dos processos.

São poucos os estudos que pesquisaram a diferença na aquisição fonológica de meninos e meninas. Alguns destes realizados com crianças da Inglaterra observaram que as meninas apresentaram melhor desempenho em algum momento do desenvolvimento fonológico ${ }^{(21-24)}$.

Pesquisas normativas, que buscam obter padrões e normas que reflitam os níveis de desempenho para grupos típicos de referência, são de grande importância, pois servem de balizadores para a tomada de decisão do fonoaudiólogo clínico em relação aos procedimentos terapêuticos a serem seguidos. No entanto, tais estudos com crianças brasileiras são escassos e geralmente realizados com um número reduzido de informantes, prejudicando a fidedignidade da padronização. Dada a dimensão continental do Brasil e a decorrente diversidade da população, é de se esperar que os dados coletados com crianças de diferentes estados sejam contrastantes, o que torna os estudos conduzidos nas diversas regiões do país relevantes para obtenção de padrões que realmente reflitam a realidade do país.

Neste sentido, o presente estudo teve como objetivo verificar o uso dos processos fonológicos em uma população de crianças com desenvolvimento fonológico normal, residentes no Rio de Janeiro. 


\section{MÉTODOS}

Foram avaliadas 240 crianças, de ambos os sexos, com idades entre três e oito anos. As crianças foram divididas em cinco grupos, de forma que cada grupo fosse composto de 48 crianças, sendo 24 meninas e 24 meninos.

Os participantes eram estudantes de duas escolas particulares do estado do Rio de Janeiro. Foram incluídas na pesquisa apenas as crianças que não apresentassem nenhuma queixa de problema auditivo, neurológico ou de fala/linguagem, de acordo com a indicação das professoras e coordenadoras pedagógicas. As crianças que estavam em tratamento fonoaudiológico foram excluídas da amostra.

Foi utilizada a avaliação FONO-FON, uma avaliação fonética e fonológica que está sendo desenvolvida pelas autoras deste artigo e que consiste em um corpus de 79 palavras apresentadas em fotografias digitais impressas em papel fotográfico, tamanho $15 \mathrm{X} 21$. As palavras selecionadas respeitam os critérios de posição do som na palavra e na estrutura silábica (onset simples inicial, onset simples medial, onset complexo inicial, onset complexo medial, coda medial e coda final) e a frequência no vocabulário infantil ${ }^{(25)}$. $\mathrm{O}$ aspecto relativo à extensão da palavra também foi contemplado na construção deste corpus, de forma que tivessem palavras monossílabas, dissílabas, trissílabas e polissílabas.

A apresentação das palavras no protocolo foi feita por sorteio de forma que a organização ficasse aleatória, para não dar pistas à criança quanto ao som inicial de cada palavra.

Esta pesquisa foi aprovada pelo Comitê de Ética em Pesquisa da Universidade Veiga de Almeida, sob número de resolução 64/06 e todos os responsáveis pelos participantes assinaram um termo de consentimento livre e esclarecido concordando com as normas do estudo.

As avaliações foram realizadas por uma pesquisadora, em sala silenciosa e com o auxílio de gravador digital (Mini Disc Sony®) para a coleta dos resultados.

A notação fonética (normas do Alfabeto Fonético Internacional - IPA 1993) foi realizada durante a avaliação e reavaliada posteriormente utilizando a gravação digital. Os dados foram confrontados com a análise de um segundo avaliador com competência na área.

Após a comparação, esclarecidas as divergências possíveis entre as duas notações, foi definida a notação final. Com base na notação final foram iniciadas as análises, onde os dados também foram confrontados com um segundo avaliador.

\section{RESULTADOS}

A análise do número de processos fonológicos foi realizada através da contagem do número de processos utilizados por cada criança. Posteriormente esses dados foram analisados e comparados por faixa etária (utilizando o teste estatístico de Kruskal-Wallis) e sexo (utilizando o teste estatístico de Mann-Whitney). A análise estatística foi realizada no programa SPSS 13.0 utilizando-se um intervalo de confiabilidade de $95 \%$.

A Tabela 1 representa o número de processos fonológicos utilizados por cada faixa etária.
Tabela 1. Números mínimo, máximo e média de processos fonológicos por faixa etária

\begin{tabular}{lcccc}
\hline Idade & Mínimo & Máximo & Média & $\begin{array}{c}\text { Desvio- } \\
\text { padrão }\end{array}$ \\
\hline 3:0 anos - 3:11 anos & 2 & 11 & 5,79 & 2,32 \\
4:0 anos - 4:11 anos & 0 & 9 & 3,64 & 2,54 \\
5:0 anos - 5:11 anos & 0 & 7 & 2,02 & 1,79 \\
6:0 anos - 6:11 anos & 0 & 3 & 0,42 & 0,71 \\
7:0 anos - 7:11 anos & 0 & 3 & 0,25 & 0,60 \\
\hline
\end{tabular}

Teste estatístico: Kruskal-Wallis. Chi-square $=153,503 / \mathrm{dF}=4 / \mathrm{p}<0,001$

De acordo com a Tabela 1 pode-se observar que a partir da faixa etária de quatro anos o mínimo de processos fonológicos utilizados foi zero e o número máximo de processos utilizados diminuiu gradativamente de acordo com o aumento da faixa etária, assim como a média e o desvio padrão. Estes resultados são estatisticamente significantes.

A Tabela 2 representa a comparação entre o número de processos fonológicos utilizados em relação ao sexo.

Tabela 2. Números mínimo, máximo e média de processos fonológicos por gênero

\begin{tabular}{lcccc}
\hline Gênero & Mínimo & Máximo & Média & $\begin{array}{c}\text { Desvio- } \\
\text { padrão }\end{array}$ \\
\hline Feminino & 0 & 11 & 2,13 & 2,55 \\
Masculino & 0 & 11 & 2,72 & 2,89 \\
\hline
\end{tabular}

Teste estatístico: Mann-Whitney. $Z=-1,416 / p=-0,157$

Como se pode observar, não houve diferença estatisticamente significante entre o número de processos fonológicos utilizados em relação ao sexo.

A análise de ocorrência de cada processo fonológico por faixa etária e sexo foi realizada buscando-se o mínimo, o máximo e a média de ocorrências de processos fonológicos por faixa etária e posteriormente por sexo.

A análise por faixa etária permite verificar que os três processos fonológicos mais utilizados na faixa etária de 3:0 a 3:11 anos são: redução de encontro consonantal, lateralização e apagamento de consoante final, com médias de ocorrência de $44,92 \%, 19,68 \%$ e $18,52 \%$, respectivamente. Os processos apócope, africação, desafricação e sonorização não foram encontrados nas crianças desta faixa etária.

$\mathrm{Na}$ faixa etária de 4:0 a 4:11 anos pode-se observar que os três processos fonológicos mais utilizados foram os mesmos da faixa etária de três anos, ou seja, redução de encontro consonantal, lateralização e apagamento de consoante final, com médias $15,23 \%, 12,39 \%$ e $6,62 \%$, respectivamente. Nesta faixa etária cinco processos fonológicos não foram utilizados: apócope, africação, sonorização, semivocalização e reduplicação.

Na faixa etária de 5:0 a 5:11 anos o processo de lateralização foi o mais utilizado, com média de 6,08\% de ocorrência seguido de redução de encontro consonantal e apagamento de consoante final, com médias de $5,14 \%$ e 3,07\%, respectivamente. Nesta faixa etária houve um aumento do número de processos não utilizados. São eles: apócope, acréscimo, 
plosivização, africação, desafricação, ensurdecimento, sonorização e reduplicação.

Na faixa etária de 6:0 a 6:11 anos o processo de redução de encontro consonantal foi o mais utilizado com média de $2,15 \%$ de ocorrência. Os demais processos tiveram médias de ocorrências em torno de $0,05 \%$, com exceção da metátese cuja média foi de $0,52 \%$, sendo o segundo processo mais utilizado. Nesta faixa etária, doze dos vinte processos fonológicos não foram utilizados, são eles: apagamento de consoante final, epêntese, apócope, acréscimo, plosivização, africação, desafricação, posteriorização, ensurdecimento, sonorização, semivocalização e reduplicação.

$\mathrm{Na}$ faixa etária de 7:0 a 7:11 anos, apenas cinco dos vinte processos fonológicos observados na pesquisa foram utilizados pelas crianças: epêntese, redução de encontro consonantal, metátese, apagamento de consoante final e assimilação, com médias de ocorrências abaixo de $0,5 \%$.

Ao observar os dados referentes à análise estatística de cada processo, verifica-se que os que obtiveram significância estatística em relação à faixa etária são: redução de encontro consonantal, apagamento de sílaba átona, apagamento de consoante final, metátese, monotongação, apagamento de líquida em onset simples, anteriorização, posteriorização, lateralização e assimilação. Os demais processos não apresentaram diferença estatisticamente significante, o que pode ser justificado pela pouca ocorrência ou ausência dos mesmos, como é o caso dos processos de apócope, africação e sonorização que não foram utilizados por nenhuma criança da pesquisa.

Em relação ao gênero, não foi observada diferença estatisticamente significante.

\section{DISCUSSÃO}

Os dados encontrados neste estudo evidenciaram que aos três anos de idade os processos de redução de encontro consonantal, lateralização e apagamento de consoante final foram os mais utilizados, sendo $44,92 \%, 19,68 \%$ e $18,52 \%$ as respectivas médias. Estes processos foram os mais utilizados também na faixa etária de quatro anos, observando-se uma diminuição gradativa nas médias de acordo com o aumento das idades. Nas crianças da faixa etária de cinco anos, observamos predominantemente processos de lateralização, redução de encontro consonantal e apagamento de consoante final.

Encontramos suporte para nossos resultados em outro estudo $^{(9)}$, com exceção dos processos de semivocalização e epêntese que não foram frequentemente observados nas crianças pesquisadas. Alguns autores ${ }^{(13)}$ também observaram que os processos fonológicos de redução de encontro consonantal, epêntese e semivocalização só desaparecem após os três anos de idade, reforçando nossas conclusões.

Em crianças falantes do inglês, de três anos a três anos e 11 meses, foi observado a presença dos processos de redução de encontro consonantal, apagamento de consoante final, apagamento de sílaba átona, anteriorização, plosivização, semivocalização e assimilação. Na faixa etária de quatro anos foram encontrados apenas os processos fonológicos de redução de encontro consonantal, apagamento de sílaba átona e semivocalização. Na faixa etária de cinco anos os processos fonológicos utilizados foram epêntese, metátese e desafricação. As crianças de seis anos em diante não fizeram uso de processos fonológicos. Semelhanças em relação à dificuldade na produção de líquidas e estruturas silábicas complexas foram observadas, uma vez que o processo de redução de encontro consonantal também neste estudo se fez presente na maior parte das crianças ${ }^{(14)}$.

Pesquisa realizada com crianças com distúrbio fonológi$\operatorname{co}^{(16)}$ encontrou muita variabilidade em relação à produção dos processos de redução de encontro consonantal e simplificação de líquidas, sendo este último, o que mais ocorreu nessa população ${ }^{(17-19)}$. Já nossos informantes de seis e sete anos demonstraram lidar de forma diferente com a dificuldade na estrutura silábica (CCV) e na produção das líquidas. Nessas faixas etárias foi observada, principalmente a presença dos processos de epêntese, redução de encontro consonantal e metátese. Este achado é corroborado por estudo ${ }^{(15)}$ que afirma que a idade prevista para a eliminação do processo de redução de encontro consonantal é aos sete anos. No que se refere à ocorrência de metátese nessa faixa etária, foi sugerido que este processo se dá majoritariamente entre as crianças mais velhas, como uma estratégia para superar dificuldades de estrutura silábica através da transposição dos componentes dessas estruturas ${ }^{(10)}$ ao invés do apagamento.

Em relação aos processos de substituição, nossos dados apontam para uma pequena porcentagem de ocorrência (menos de $2 \%$ aos três anos), de forma a não serem considerados como característicos do desenvolvimento fonológico das crianças estudadas, não concordando com outros estudos sobre os processos fonológicos de substituição ${ }^{(11-12)}$. Não foi observada nas crianças pesquisadas a ocorrência do processo de ensurdecimento como observado em crianças com transtorno fonológico ${ }^{(18)}$.

Em relação ao número de processos fonológicos encontrados por faixa etária, observou-se que aos três anos de idade, as crianças utilizaram um mínimo de dois de processos fonológicos, sendo a média 5,79. A partir da faixa etária de quatro anos o número mínimo de processos fonológicos utilizados estabilizou-se em zero e o número máximo diminuiu gradativamente de acordo com o aumento da faixa etária, assim como a média. Não encontramos dados similares na literatura para a discussão deste aspecto.

Em relação à variável gênero, não foi observada nenhuma diferença estatisticamente significante em nenhuma das análises realizadas nesta pesquisa.

Estes dados não estão em concordância com estudos ${ }^{(21-24)}$ que observaram que as meninas apresentaram melhor desempenho em algum momento do desenvolvimento fonológico.

\section{CONCLUSÃO}

Os dados permitem concluir que aos três anos de idade os processos fonológicos mais utilizados foram: redução de encontro consonantal, lateralização e apagamento de consoante final. Estes processos foram os mais utilizados também na faixa etária de quatro anos, tendo uma diminuição significativa das médias de acordo com o aumento da idade. Já na faixa etária de cinco anos observou-se maior frequência do processo de 
lateralização, seguido de redução de encontro consonantal e apagamento de consoante final. Aos seis anos, o processo de redução de encontro consonantal foi novamente o mais utilizado seguido da metátese. Já na faixa etária de sete anos, a epêntese foi o processo fonológico que mais ocorreu, ficando a redução de encontro consonantal e metátese em segundo e terceiro lugares.

Em relação à análise referente ao número de processos fonológicos encontrados por faixa etária, observamos que aos três anos de idade, as crianças utilizaram um mínimo de dois de processos fonológicos. A partir da faixa etária de quatro anos o número mínimo de processos fonológicos utilizados estabilizouse em zero e o número máximo diminuiu gradativamente de acordo com o aumento da faixa etária, assim como a média.

Em relação à variável gênero, não foi observada nenhuma diferença estatisticamente significante em nenhuma das análises realizadas nesta pesquisa.

Os dados encontrados evidenciam a dificuldade encontrada pelas crianças na produção das líquidas e nas estruturas silábicas mais complexas.

\begin{abstract}
Purpose: The aim of this study was to verify the use of phonological processes in a group of children with normal phonological development. Methods: The participants were 240 children of both genders, aged between three and eight years. Analyses regarding phonological processes were carried out, and the data were compared considering age and gender. Results: The results allowed the conclusion that at the ages of three, four and five years the most frequently used processes were cluster reduction, lateralization, and final consonant deletion. Metathesis was the second most frequently used process at the age of six, and the third at the age of seven. Concerning the number of phonological processes used at each age range, three-year-old children used at least two processes, and from the age of four onward the minimum number of processes dropped to zero, while the maximum number and the mean number of processes gradually decreased with increasing age. Regarding gender, not a single significant difference was found for any of the analyses performed in the current study. Conclusions: The data obtained in this study evidenced the difficulties found by normally developing children in the production of liquid phonemes and complex syllabic structures.
\end{abstract}

Keywords: Child language; Evaluation; Child development; Language development

\section{REFERÊNCIAS}

1. Keske-Soares M, Blanco APF, Mota HB. O desvio fonológico caracterizado por índices de substituição e omissão. Rev Soc Bras Fonoaudiol. 2004;9(1):10-8.

2. Pereira LF, Mota HB. Tratamento fonológico baseado nos contrastes de oposições máximas. Pró-Fono. 2002;14(2):165-74.

3. Wertzner HF. Fonologia: desenvolvimento e alterações. In: Ferreira LP, Befi-Lopes DM, Limongi SCO. Tratado de fonoaudiologia. São Paulo: Roca; 2004. p. 772-86.

4. Lowe RJ. Fonologia: avaliação e intervenção: aplicações na patologia da fala. Porto Alegre: Artes Médicas; 1996.

5. Andrade CRF. Prevalência das desordens idiopáticas da fala e da linguagem em crianças de um a onze anos de idade. Rev Saúde Pública = J Public Health. 1997;31(5):495-501.

6. Magalhães JS. A metátese da líquida não-lateral na aquisição: evidências para o pé troqueu. Letras Hoje. 2003;38(2): 83-95

7. Van Borsel J. Kinderen met spraakproductieproblemen. Fonologische procesanalyse met oefeningen. Leuven/Leusen: Acco; 2003.

8. Gonçalves CS. O processo de assimilação na assimilação fonológica. Pró-Fono. 2002;14(3):291-300.

9. Ribas LP. Onset complexo: características da aquisição. Letras Hoje. 2003;38(2): 23-31.

10. Lamprecht RR. Perfil da aquis ição da fonologia do Português [tese]. Porto Alegre: Pontifícia Universidade Católica do Rio Grande do Sul; 1990.

11. Lamprecht RR. Aquisição fonológica do português: perfil de desenvolvimento e subsídios para terapia. Porto Alegre: Artmed; 2004.

12. Oliveira CC. Perfil da aquisição das fricativas /f/, /v/, /Š /, /Ž/ do Português Brasileiro: um estudo quantitativo. Letras Hoje. 2003;38(2):97-110.

13. Peña-Brooks A, Hedge MN. Assessment and treatment of articulation and phonological disorders in children. A dual-level text. Austin, Texas: Pro Ed; 2000.
14. McLeod S, Bleile K. Neurological and developmental foundations of speech acquisition. American Speech Language-Hearing Association Convention. Chicago: ASHA; 2003.

15. Andrade CRF, Befi-Lopes DM, Fernandes FDM, Wertzner HF. ABFW: teste de linguagem infantil nas áreas de fonologia, vocabulário, fluência e pragmática. Barueri: Pró-Fono; 2000.

16. Wertzner HF, Oliveira MMF. Semelhanças entre os sujeitos com distúrbio fonológico. Pró-Fono. 2002;14(2)143-52.

17. Papp ACCS, Wertzner HF. O aspecto familial e o transtorno fonológico. Pró-Fono. 2006;18(2):151-60.

18. Wertzner HF, Pagan LO, Galea DES, Papp ACCS. Características fonológicas de crianças com transtorno fonológico com e sem histórico de otite média. Rev Soc Bras Fonoaudiol. 2007;12(1):41-7.

19. Wertzner HF, Papp ACCS, Galea DES. Provas de nomeação e imitação como instrumentos de diagnóstico do transtorno fonológico. Pró-Fono. 2006;18(3):303-12.

20. Lima LM, Queiroga BAM. Aquisição fonológica em crianças com antecedentes de desnutrição. Rev CEFAC. 2007;9(1):13-20.

21. Dodd B, Holm A, Hua Z, Crosbie S. Phonological development: a normative study of British English-speaking children. Clin Linguist Phon. 2003;17(8):617-43.

22. Wellman B, Case I, Mengert I, Bradbury D. Speech sounds of young children. University of Iowa Study, Child Welfare.1931;5(2).

23. Poole I. Genetic development of articulation of consonant sounds in speech. Elem Eng Rev. 1934;11:159-61.

24. Smit AB, Hand L, Freilinger JJ, Bernthal JE, Bird A. The Iowa Articulation Norms Project and its Nebraska replication. J Speech Hear Disord. 1990;55(4):779-98. Erratum in: J Speech Hear Disord. 1991;34(2):446.

25. Pinheiro AMV. Leitura e escrita: Uma abordagem cognitiva. São Paulo: Editora Psy Ii;1994. 